\title{
The MIMO Wiretap Channel
}

\author{
Frédérique Oggier and Babak Hassibi \\ Department of Electrical Engineering, \\ California Institute of Technology, \\ Pasadena 91125 CA, USA. \\ Email:\{frederique, hassibi\}asystems.caltech.edu
}

\begin{abstract}
We study the MIMO wiretap channel, a MIMO broadcast channel where the transmitter sends some confidential information to one user which is a legitimate receiver, while the other user is an eavesdropper. Perfect secrecy is achieved when the transmitter and the legitimate receiver can communicate at some positive rate, while ensuring that the eavesdropper gets zero bits of information. In this paper, we compute the perfect secrecy capacity of the multiple antenna MIMO broadcast channel, where the number of antennas is arbitrary for both the transmitter and the two receivers. Our technique involves a careful study of a Sato-like upper bound via the solution of a certain algebraic Riccati equation.
\end{abstract}

\section{INTRODUCTION}

In the traditional confidentiality setting, a transmitter (Alice) wants to send some secret message to a legitimate receiver (Bob), and prevent the eavesdropper (Eve) from reading the message.

From an information theoretic point of view, the communication channel involved can be modeled as a broadcast channel, following the wire-tap channel model introduced by Wyner [22]: a transmitter broadcasts its message, say $w^{k} \in \mathcal{W}^{k}$, encoded into a codeword $x^{n}$, and the two receivers (the legitimate and the illegitimate) respectively receive $y^{n}$ and $z^{n}$, the output of their channels. The amount of ignorance that the eavesdropper has about a message $w^{k}$ is called the equivocation rate, defined as:

Definition 1: The equivocation rate $R_{e}$ is defined as

$$
R_{e}=\frac{1}{n} h\left(w^{k} \mid z^{n}\right),
$$

with $0 \leq R_{e} \leq h\left(w^{k}\right) / n$. Clearly, if $R_{e}$ is equal to the information rate $h\left(w^{k}\right) / n$, then $I\left(z^{n} \mid w^{k}\right)=0$, which yields perfect secrecy.

Associated with secrecy is a perfect secrecy rate $R_{s}$, which is the amount of information that can be sent not only reliably but also confidentially, with the help of a $\left(2^{n R_{s}}, n\right)$ code.

Definition 2: A perfect secrecy rate $R_{s}$ is said to be achievable if for any $\epsilon, \epsilon^{\prime}>0$, there exists a sequence of $\left(2^{n R_{s}}, n\right)$ codes such that for any $n \geq n\left(\epsilon, \epsilon^{\prime}\right)$, we have

$$
\begin{aligned}
P_{e} & \leq \epsilon^{\prime} \\
R_{s}-\epsilon & \leq R_{e} .
\end{aligned}
$$

${ }^{1}$ This work was supported in part by the National Science Foundation under grant CCF-0729203, by the David and Lucille Packard Foundation and by Caltech's Lee Center for Advanced Networking.
The first condition (1), where $P_{e}$ is the probability of decoding erroneously, is the standard definition of achievable rate as far as reliability is concerned. The second condition (2) guarantees secrecy, up to the equivocation rate, which we will require to be $h\left(w^{k}\right) / n$ to have perfect secrecy. The secrecy capacity is defined similarly to the standard capacity:

Definition 3: The secrecy capacity $C_{s}$ is the maximum achievable perfect secrecy rate.

In this paper, we are interested in the secrecy capacity for the case where Alice, Bob and Eve are communicating via multiple antenna channels.

\section{A. Previous work}

In his seminal work [22], Wyner showed for discrete memoryless channels that the perfect secrecy capacity is actually the difference of the capacity of the two users, under the assumption that the channel of the eavesdropper is a degraded version of the channel of the legitimate receiver. This result has been generalized to Gaussian channels by Leung et al. [9].

In [4], Gopala et al. have shown that the secrecy capacity is also the difference of the two capacities in the case of a single antenna fading channel, under the assumption of asymptotically long coherence intervals, when the transmitter either knows both channels or only the legitimate channel. In [1], [2], Barros et al. have characterized information theoretic security in terms of outage probability. Independently, Liang et al. [12], [13] and Li et al. [10] have computed the secrecy capacity for the parallel wiretap channel with independent subchannels. The secrecy capacity of the wiretap channel with single antenna fading channel follows.

A first study involving multiple antenna channels has been proposed by Hero [5], in a different context than the wiretap channel. In [19], the SIMO wiretap channel has been considered. In [11], the secrecy capacity is computed for the MISO case. Furthermore, a lower bound is computed in the MIMO case. The secrecy capacity for the MISO case has also been proven independently by Khisti et al. [7] and Shafiee et al. [20]. In [7], the authors furthermore give an upper bound for the MIMO case, in a regime asymptotic in SNR. The secrecy capacity has been computing for the particular cases where both the transmitter and receiver have two antennas, and the eavesdropper has either one antenna [21] or two antennas [17]. Finally, Liu et al. [14], [15] computed the secrecy capacity for a Gaussian broadcast channel, where a multiantenna transmitter sends independent confidential messages 

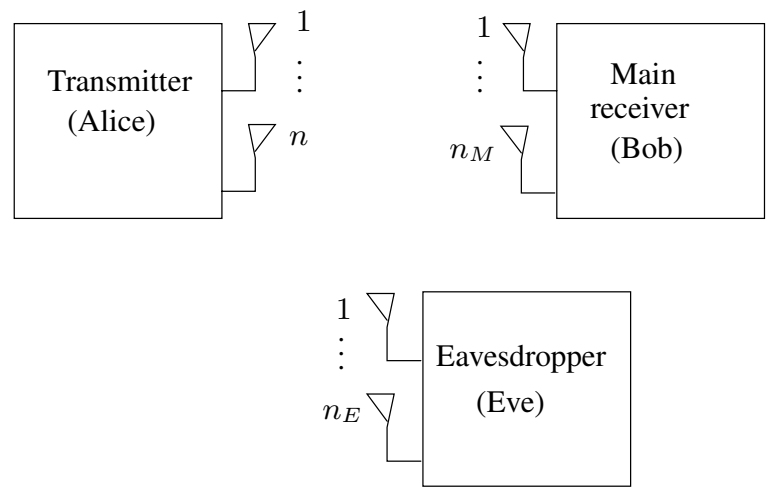

Fig. 1. The MIMO wiretap channel

to two users.

The contribution of this paper is to compute the perfect secrecy capacity of the multiple antenna wire-tap channel, for any number of transmit/receive antennas. In order to compute the secrecy capacity, we provide a proof technique for the converse, which allows us to deal with channels that are not degraded. Note that our result shows that the inner bound by Li et al. [11] is tight, and this is proved by the computation of an upper bound that actually matches the lower bound. Independently of our results, Khisthi and Wornell [8] have also computed the secrecy capacity of the MIMO wiretap channel (which they refer to as MIMO-ME). An alternative derivation of our result, and that of Khisti-Wornell, has also appeared in Liu and Shamai [16].

\section{B. The MIMO wiretap channel}

We consider the MIMO wiretap channel, that is, a broadcast channel where the transmitter is equipped with $n$ transmit antennas, while the legitimate receiver and an eavesdropper have respectively $n_{M}$ and

$n_{E}$ receive antennas, namely:

$$
\begin{aligned}
Y & =H_{M} X+V_{M} \\
Z & =H_{E} X+V_{E}
\end{aligned}
$$

where $Y, V_{M}$ and $Z, V_{E}$ are resp. $n_{M} \times 1$ and $n_{E} \times 1$ vectors. We have that $X$ is the $n \times 1$ complex transmitted signal, with covariance matrix $K_{X} \succeq \mathbf{0}_{n}$ with power constraint $\operatorname{Tr}\left(K_{X}\right)=P$, while $H_{M}$ and $H_{E}$ are respectively $n_{M} \times n$ and $n_{E} \times n$ fixed channel matrices. They are both assumed to be known at the transmitter. Along the paper we will usually consider two cases: the definite case, that is when $H_{M}^{*} H_{M} \succ H_{E}^{*} H_{E}$ or $H_{E}^{*} H_{E} \succ H_{M}^{*} H_{M}$, which corresponds to the degraded case, and the indefinite case, which is when some of the eigenvalues of $H_{E}^{*} H_{E}-H_{M}^{*} H_{M}$ are positive, and other negative or zero. The vectors $V_{M}, V_{E}$ are independent circularly symmetric complex Gaussian with identity covariance $K_{M}=\mathbf{I}_{n_{M}}, K_{E}=\mathbf{I}_{n_{E}}$ and independent of the transmitted signal $X$.

Our main result is:
Theorem 1: The secrecy capacity $C_{S}$ of the MIMO wiretap channel is given by

$$
\max _{K_{X} \succeq \mathbf{0}} \log \operatorname{det}\left(\mathbf{I}+H_{M} K_{X} H_{M}^{*}\right)-\log \operatorname{det}\left(\mathbf{I}+H_{E} K_{X} H_{E}^{*}\right)
$$

with $\operatorname{Tr}\left(K_{X}\right)=P$.

The paper contains the main parts of the proof of the above theorem.

\section{ON THE ACHIEVABILITY}

In this section, we state the achievability part of the secrecy capacity, and further prove that in the non-degraded case, the achievability is maximized by $n \times n$ matrices $K_{X}$ which are low rank, that is of any rank $r<n$.

Proposition 1: The perfect secrecy rate

$$
R_{s}=\max _{K_{X} \succeq \mathbf{0}} \log \operatorname{det}\left(\mathbf{I}+H_{M} K_{X} H_{M}^{*}\right)-\log \operatorname{det}\left(\mathbf{I}+H_{E} K_{X} H_{E}^{*}\right)
$$

with $\operatorname{Tr}\left(K_{X}\right)=P$, is achievable.

This has already been proved [11]. In fact, the interpretation is obvious. When $K_{X}$ is chosen, the difference between the resulting mutual informations to the legitimate user and eavesdropper can be secretly transmitted.

Proposition 2: Let $\tilde{K}_{X}$ be an optimal solution to the optimization problem

$$
\max _{K_{X} \succeq \mathbf{0}} \log \operatorname{det}\left(\mathbf{I}+H_{M} K_{X} H_{M}^{*}\right)-\log \operatorname{det}\left(\mathbf{I}+H_{E} K_{X} H_{E}^{*}\right),
$$

where $\operatorname{Tr}\left(K_{X}\right)=P$ and $H_{E}^{*} H_{E}-H_{M}^{*} H_{M}$ is either indefinite or semidefinite. Then $\tilde{K}_{X}$ is a low rank matrix.

Proof: To show that the optimal $\tilde{K}_{X}$ is low rank, we define a Lagrangian which includes the power constraint, and show that this yields no solution. From there, we can conclude that the optimal solution is on the boundary of the cone of positive semi-definite matrices, i.e., matrices of rank $r<n$.

We thus define the following Lagrangian:

$$
\begin{gathered}
\log \operatorname{det}\left(\mathbf{I}_{n_{M}}+H_{M} K_{X} H_{M}^{*}\right) \\
-\log \operatorname{det}\left(\mathbf{I}_{n_{E}}+H_{E} K_{X} H_{E}^{*}\right)-\lambda \operatorname{Tr}\left(K_{X}\right),
\end{gathered}
$$

and look for its stationary points, that is for the solution of the following equation:

$$
\begin{gathered}
\nabla_{K_{X}}\left(\log \operatorname{det}\left(\mathbf{I}+H_{M} K_{X} H_{M}^{*}\right)\right. \\
\left.-\log \operatorname{det}\left(\mathbf{I}+H_{E} K_{X} H_{E}^{*}\right)-\lambda \operatorname{Tr}\left(K_{X}\right)\right)=0 \\
\Longleftrightarrow H_{M}^{*} H_{M}\left(\mathbf{I}+K_{X} H_{M}^{*} H_{M}\right)^{-1} \\
=\left(\mathbf{I}+H_{E}^{*} H_{E} K_{X}\right)^{-1} H_{E}^{*} H_{E}+\lambda \mathbf{I}_{n} .
\end{gathered}
$$

By pre-multiplying the above equation by $\left(\mathbf{I}+H_{E}^{*} H_{E} K_{X}\right)$ and post-multiplying it by $\left(\mathbf{I}+K_{X} H_{M}^{*} H_{M}\right)$, we get

$$
H_{M}^{*} H_{M}-H_{E}^{*} H_{E}=\lambda\left(\mathbf{I}+H_{E}^{*} H_{E} K_{X}\right)\left(\mathbf{I}+K_{X} H_{M}^{*} H_{M}\right),
$$

or equivalently, by further pre and post-multiplying by $K_{X}$,

$$
\begin{gathered}
K_{X}\left(H_{M}^{*} H_{M}-H_{E}^{*} H_{E}\right) K_{X} \frac{1}{\lambda}= \\
\left(K_{X}+K_{X} H_{E}^{*} H_{E} K_{X}\right)\left(K_{X}+K_{X} H_{M}^{*} H_{M} K_{X}\right) .
\end{gathered}
$$

Now if $K_{X} \succ \mathbf{0}$, then all the eigenvalues of $\left(K_{X}+\right.$ $\left.K_{X} H_{E}^{*} H_{E} K_{X}\right)\left(K_{X}+K_{X} H_{M}^{*} H_{M} K_{X}\right)$ are strictly positive (Lemma 1 below). This implies that (4) can have a solution if and only if the Hermitian matrix $K_{X}\left(H_{M}^{*} H_{M}-H_{E}^{*} H_{E}\right) K_{X} \frac{1}{\lambda}$ 
is positive definite. This means that either $H_{M}^{*} H_{M} \succ H_{E}^{*} H_{E}$ and $\lambda>0$, or $H_{M}^{*} H_{M} \prec H_{E}^{*} H_{E}$ and $\lambda<0$. This gives a contradiction if $H_{M}^{*} H_{M}-H_{E}^{*} H_{E}$ is either indefinite or semidefinite, implying that $\tilde{K}_{X}$ has to be low rank.

Lemma 1: If $A=A^{*} \succ \mathbf{0}$ and $B=B^{*} \succ \mathbf{0}$, then the matrix $A B$ has all positive eigenvalues.

Proof: Since $A \succ \mathbf{0}$, we can write $A=A^{1 / 2}\left(A^{*}\right)^{1 / 2}$ with $A^{1 / 2}$ invertible. Therefore,

$$
A B=A^{1 / 2}\left(\left(A^{*}\right)^{1 / 2} B A^{1 / 2}\right) A^{-1 / 2},
$$

has the same eigenvalues as the matrix $\left(A^{*}\right)^{1 / 2} B A^{1 / 2}$, which is positive definite.

\section{Proof OF THE CONVERSE}

The goal of this section is to prove the converse, namely

Theorem 2: For any sequence of $\left(2^{n R_{s}}, n\right)$ codes with probability of error $P_{e} \leq \epsilon^{\prime}$ and equivocation rate $R_{s}-\epsilon \leq R_{e}$ for any $n \geq n\left(\epsilon, \epsilon^{\prime}\right), \epsilon, \epsilon^{\prime}>0$, then the secrecy rate $R_{s}$ satisfies $R_{s} \leq \max _{K_{X} \succeq \mathbf{0}} \log \operatorname{det}\left(\mathbf{I}+H_{M} K_{X} H_{M}^{*}\right)-\log \operatorname{det}\left(\mathbf{I}+H_{E} K_{X} H_{E}^{*}\right)$, with $\operatorname{Tr}\left(K_{X}\right)=P$.

\section{A. Bound on $I(X ; Y \mid Z)$ and result for the degraded case}

We start by recalling a standard result [9], [4].

Lemma 2: Given any sequence of $\left(2^{n R_{s}}, n\right)$ codes with $P_{e} \leq \epsilon$ and $R_{s}-\epsilon \leq R_{e}$ for any $n \geq n(\epsilon), \epsilon>0$, the secrecy rate $R_{s}$ can be upper bounded as follows:

$$
R_{s}-\epsilon \leq \frac{1}{n}\left[I\left(\left(X^{n}, Y^{n} \mid Z^{n}\right)+\delta\right], \epsilon, \delta>0 .\right.
$$

We thus focus on finding an upper bound on $I(X ; Y \mid Z)$. Proposition 3: We have the following upper bound:

$$
I(X ; Y \mid Z) \leq \max _{K_{X} \succeq \mathbf{0}} \tilde{I}(X ; Y \mid Z)
$$

where $\tilde{I}(X ; Y \mid Z)$ is given by

$$
\begin{gathered}
\log \operatorname{det}\left(\mathbf{I}+\left(\begin{array}{ll}
H_{M}^{*} & H_{E}^{*}
\end{array}\right)\left(\begin{array}{cc}
\mathbf{I} & A \\
A^{*} & \mathbf{I}
\end{array}\right)^{-1}\left(\begin{array}{c}
H_{M} \\
H_{E}
\end{array}\right) K_{X}\right) \\
-\log \operatorname{det}\left(\mathbf{I}+H_{E} K_{X} H_{E}^{*}\right)
\end{gathered}
$$

and $A$ denotes the correlation between $V_{M}$ and $V_{E}$, which satisfies $\mathbf{I}-A A^{*} \succ \mathbf{0}$.

Proof: An upper bound on $I(X ; Y \mid Z)$ is obtained by assuming that the legitimate receiver knows both its channel and the one of the eavesdropper, so that the capacity of the link between the transmitter and the legitimate receiver is that of a MIMO system, namely

$\max _{K_{X}} \log \operatorname{det}\left[\mathbf{I}_{n}+\left[\begin{array}{ll}H_{M}^{*} & H_{E}^{*}\end{array}\right]\left[\begin{array}{cc}\mathbf{I}_{n_{M}} & A \\ A^{*} & \mathbf{I}_{n_{E}}\end{array}\right]^{-1}\left[\begin{array}{c}H_{M} \\ H_{E}\end{array}\right] K_{X}\right]$

where $A$ has to satisfy $\mathbf{I}-A A^{*} \succ \mathbf{0}$. Now the channel we consider is degraded, and an upper bound is thus the difference of the two capacities, which yields the result.

We can now conclude the proof of the converse for the "simple" cases when $H_{M}^{*} H_{M} \succ H_{E}^{*} H_{E}$ or $H_{E}^{*} H_{E} \succ H_{M}^{*} H_{M}$.
Proposition 4: 1) If $H_{M}^{*} H_{M} \succ H_{E}^{*} H_{E}$, we have that

$$
\begin{aligned}
I(X ; Y \mid Z) \leq & \max _{K_{X} \succeq \mathbf{0}} \log \operatorname{det}\left(\mathbf{I}+H_{M} K_{X} H_{M}^{*}\right)- \\
& \log \operatorname{det}\left(\mathbf{I}+H_{E} K_{X} H_{E}^{*}\right) .
\end{aligned}
$$

2) Vice versa, if $H_{E}^{*} H_{E} \succ H_{M}^{*} H_{M}$, then $I(X ; Y \mid Z)=0$.

Proof: Let us introduce two other ways of writing $\tilde{I}(X ; Y \mid Z)$ (see (5)). Let us first compute a UDL factorization:

$$
\left[\begin{array}{cc}
\mathbf{I}_{n_{M}} & A \\
A^{*} & \mathbf{I}_{n_{E}}
\end{array}\right]=\left[\begin{array}{cc}
\mathbf{I} & A \\
\mathbf{0} & \mathbf{I}
\end{array}\right]\left[\begin{array}{cc}
\mathbf{I}-A A^{*} & \mathbf{0} \\
\mathbf{0} & \mathbf{I}
\end{array}\right]\left[\begin{array}{cc}
\mathbf{I} & \mathbf{0} \\
A^{*} & \mathbf{I}
\end{array}\right]
$$

so that

$$
\left[\begin{array}{cc}
\mathbf{I} & A \\
A^{*} & \mathbf{I}
\end{array}\right]^{-1}=\left[\begin{array}{cc}
\mathbf{I} & \mathbf{0} \\
-A^{*} & \mathbf{I}
\end{array}\right]\left[\begin{array}{cc}
\left(\mathbf{I}-A A^{*}\right)^{-1} & \mathbf{0} \\
\mathbf{0} & \mathbf{I}
\end{array}\right]\left[\begin{array}{cc}
\mathbf{I} & -A \\
\mathbf{0} & \mathbf{I}
\end{array}\right]
$$

and we have that

$$
\begin{gathered}
\left(\begin{array}{ll}
H_{M}^{*} & H_{E}^{*}
\end{array}\right)\left(\begin{array}{cc}
\mathbf{I} & A \\
A^{*} & \mathbf{I}
\end{array}\right)^{-1}\left(\begin{array}{c}
H_{M} \\
H_{E}
\end{array}\right)= \\
\left(H_{M}^{*}-H_{E}^{*} A^{*}\right)\left(\mathbf{I}-A A^{*}\right)^{-1}\left(H_{M}-A H_{E}\right)+H_{E}^{*} H_{E} .
\end{gathered}
$$

Thus a first equivalent formula for $\tilde{I}(X ; Y \mid Z)$ is given by

$$
\begin{gathered}
\log \operatorname{det}\left(\mathbf{I}+\left(\left(H_{M}^{*}-H_{E}^{*} A^{*}\right)\left(\mathbf{I}-A A^{*}\right)^{-1}\left(H_{M}-A H_{E}\right)+\right.\right. \\
\left.\left.H_{E}^{*} H_{E}\right) K_{X}\right)-\log \operatorname{det}\left(\mathbf{I}+H_{E} K_{X} H_{E}^{*}\right) .
\end{gathered}
$$

By considering now a LDU factorization, we get

$$
\begin{gathered}
{\left[\begin{array}{cc}
\mathbf{I} & A \\
A^{*} & \mathbf{I}
\end{array}\right]=\left[\begin{array}{cc}
\mathbf{I} & \mathbf{0} \\
A^{*} & \mathbf{I}
\end{array}\right]\left[\begin{array}{cc}
\mathbf{I} & \mathbf{0} \\
\mathbf{0} & \mathbf{I}-A^{*} A
\end{array}\right]\left[\begin{array}{cc}
\mathbf{I} & A \\
\mathbf{0} & \mathbf{I}
\end{array}\right],} \\
{\left[\begin{array}{cc}
\mathbf{I} & A \\
A^{*} & \mathbf{I}
\end{array}\right]^{-1}=\left[\begin{array}{cc}
\mathbf{I} & -A \\
\mathbf{0} & \mathbf{I}
\end{array}\right]\left[\begin{array}{cc}
\mathbf{I} & \mathbf{0} \\
\mathbf{0} & \left(\mathbf{I}-A^{*} A\right)^{-1}
\end{array}\right]\left[\begin{array}{cc}
\mathbf{I} & \mathbf{0} \\
-A^{*} & \mathbf{I}
\end{array}\right]}
\end{gathered}
$$

so that

$$
\begin{gathered}
\left(\begin{array}{ll}
H_{M}^{*} & H_{E}^{*}
\end{array}\right)\left(\begin{array}{cc}
\mathbf{I} & A \\
A^{*} & \mathbf{I}
\end{array}\right)^{-1}\left(\begin{array}{c}
H_{M} \\
H_{E}
\end{array}\right)=H_{M}^{*} H_{M}+ \\
\left(-H_{M}^{*} A+H_{E}^{*}\right)\left(\mathbf{I}-A^{*} A\right)^{-1}\left(-A^{*} H_{M}+H_{E}\right)
\end{gathered}
$$

and a second equivalent formula for $\tilde{I}(X ; Y \mid Z)$ is given by

$$
\begin{gathered}
\log \operatorname{det}\left(\mathbf{I}+H_{M}^{*} H_{M} K_{X}+\right. \\
\left.\left(-H_{M}^{*} A+H_{E}^{*}\right)\left(\mathbf{I}-A^{*} A\right)^{-1}\left(-A^{*} H_{M}+H_{E}\right) K_{X}\right) \\
-\log \operatorname{det}\left(\mathbf{I}+H_{E} K_{X} H_{E}^{*}\right) .
\end{gathered}
$$

Since the secrecy capacity does not depend on $A$, and that

$$
I(X ; Y \mid Z) \leq \max _{K_{X}} \tilde{I}(X ; Y \mid Z),
$$

for all $A$ such that $\mathbf{I}-A A^{*} \succ \mathbf{0}$, we are now free to take any such $A$ which does not depend on a choice of $K_{X}$.

Case 1. If $H_{M}^{*} H_{M} \succ H_{E}^{*} H_{E}$, we will now show that there always exists

a matrix $A$ such that $H_{M}^{*} A=H_{E}^{*}$ and $\mathbf{I}-A A^{*} \succ \mathbf{0}$. Note that using (7), we then get

$\tilde{I}(X ; Y \mid Z)=\log \operatorname{det}\left(\mathbf{I}+H_{M}^{*} H_{M} K_{X}\right)-\log \operatorname{det}\left(\mathbf{I}+H_{E}^{*} H_{E} K_{X}\right)$.

Now $H_{M}^{*} H_{M} \succ H_{E}^{*} H_{E}$ implies that $H_{M} H_{M}^{*}=H_{E}^{*} H_{E}+$ $X^{*} X$, for some $X^{*} X \succ 0$. Now this means [6] that there 
exists a unitary matrix $\Theta$ such that $\left[\begin{array}{ll}H_{E}^{*} & X^{*}\end{array}\right]=\left[\begin{array}{ll}H_{M}^{*} & \mathbf{0}\end{array}\right] \Theta$. Partitioning $\Theta$, we get

$$
\left[\begin{array}{ll}
H_{E}^{*} & X^{*}
\end{array}\right]=\left[\begin{array}{ll}
H_{M}^{*} & \mathbf{0}
\end{array}\right]\left[\begin{array}{ll}
\Theta_{11} & \Theta_{12} \\
\Theta_{21} & \Theta_{22}
\end{array}\right]
$$

from which it follows that $H_{E}^{*}=H_{M}^{*} \Theta_{11}$. Note that we can take $A=\Theta_{11}$, since $\Theta_{11}^{*} \Theta_{11} \prec \mathbf{I}$ as it is a sub-block of a unitary matrix, and using the fact that $X^{*} X \succ \mathbf{0}$.

Case 2. This is similar when $H_{E}^{*} H_{E} \succ H_{M}^{*} H_{M}$.

The cases described in the lemma can be understood as a simple generalization of the scalar case, since those are the degraded cases. When $H_{M}^{*} H_{M} \succ H_{E}^{*} H_{E}$, all links to the legitimate receiver are better, and the capacity is given by the difference of the two capacities, while if $H_{E}^{*} H_{E} \succ H_{M}^{*} H_{M}$, then all links to the eavesdropper are better, and thus no positive secrecy capacity can be achieved.

We are now left with the interesting case when $H_{M}^{*} H_{M}-$ $H_{E}^{*} H_{E}$ is indefinite, which is the non-degraded case.

\section{B. Minimization over $A$ and maximization over $K_{X}$}

Since Proposition 3 is true for all $A$ such that $\mathbf{I}-A A^{*} \succ \mathbf{0}$, we get

$$
I(X ; Y \mid Z) \leq \min _{A} \max _{K_{X}} \tilde{I}(X ; Y, Z) .
$$

To understand this double optimization, we start by analyzing the function $\tilde{I}(X ; Y, Z)$.

Proposition 5: The function $\tilde{I}(X ; Y, Z)$ defined in (5) is concave in $K_{X}$ and convex in $A$. Consequently,

$$
\min _{A} \max _{K_{X}} \tilde{I}(X ; Y \mid Z)=\max _{K_{X}} \min _{A} \tilde{I}(X ; Y \mid Z)
$$

where $\operatorname{Tr}\left(K_{X}\right)=P, K_{X} \succeq \mathbf{0}, \mathbf{I}-A A^{*} \succ \mathbf{0}$.

This proof is skipped here by lack of space (see [18]).

We next compute the minimization over $A$. Note that we can write $\tilde{I}(X ; Y \mid Z)$ in the following alternative way:

$$
\begin{gathered}
\log \operatorname{det}\left(H_{M} K_{X} H_{M}^{*}+\mathbf{I}_{n_{M}}-\right. \\
\left.\left(H_{M} K_{X} H_{E}^{*}+A\right)\left(H_{E} K_{X} H_{E}^{*}+\mathbf{I}\right)^{-1}\left(H_{E} K_{X} H_{M}^{*}+A^{*}\right)\right) \\
-\log \operatorname{det}\left(\mathbf{I}_{n_{M}}-A A^{*}\right)
\end{gathered}
$$

Proposition 6: Let $\tilde{A}^{*}$ be a local minima of $\tilde{I}(X ; Y \mid Z)$. Then

$$
\tilde{A}^{*}=\left(\begin{array}{ll}
H_{E} V & Q W
\end{array}\right)\left(H_{M} V \quad P W\right)^{-1},
$$

where $W$ is an $m_{1} \times m_{2}$ matrix, $0 \leq m_{2} \leq n_{M},\left(P^{T} Q^{T}\right)^{T}$ is orthogonal to $\left(-H_{M}^{*} H_{E}^{*}\right), P, Q$ of dimension resp. $n_{M} \times m_{1}$, $n_{e} \times m_{1}$, and $V$ is a $n \times\left(n_{M}-m_{2}\right)$ matrix, such that

$$
\left(\begin{array}{c}
H_{M} V \\
H_{E} V
\end{array}\right)
$$

is an invariant subspace of $M$, as defined in (9).

Proof: Let $M_{1}, M_{2}, M_{3}, X$ be square complex matrices. Set $f(X)=M_{1}-\left(X+M_{2}\right) M_{3}\left(X^{*}+M_{2}^{*}\right)$. We have that

$$
\nabla_{X} \log \operatorname{det}(f(X))=-f(X)^{-1}\left(X+M_{2}\right) M_{3} .
$$

Using this formula, we compute that $\nabla_{A^{*}} \tilde{I}(X ; Y \mid Z)=0$ iff

$$
\begin{gathered}
f(A)\left(A^{*}+H_{E} K_{X} H_{M}^{*}\right)^{-1}\left(H_{E} K_{X} H_{E}^{*}+\mathbf{I}\right)= \\
\left(\mathbf{I}-A A^{*}\right)\left(A^{*}\right)^{-1},
\end{gathered}
$$

where $f(A)$ is given by

$$
\begin{gathered}
H_{M} K_{X} H_{M}^{*}+\mathbf{I} \\
-\left(H_{M} K_{X} H_{E}^{*}+A\right)\left(H_{E} K_{X} H_{E}^{*}+\mathbf{I}\right)^{-1}\left(H_{E} K_{X} H_{M}^{*}+A^{*}\right) .
\end{gathered}
$$

We get a nonsymmetric algebraic Ricatti equation given by

$$
\begin{gathered}
A^{*}\left(H_{M} K_{X} H_{M}^{*}+\mathbf{I}\right)^{-1} H_{M} K_{X} H_{E}^{*} A^{*}+A^{*}\left(H_{M} K_{X} H_{M}^{*}+\mathbf{I}\right)^{-1}+ \\
{\left[-H_{E} K_{X} H_{E}^{*}-\mathbf{I}+H_{E} K_{X} H_{M}^{*}\left(H_{M} K_{X} H_{M}^{*}+\mathbf{I}\right)^{-1} H_{M} K_{X} H_{E}^{*}\right] A^{*}} \\
+H_{E} K_{X} H_{M}^{*}\left(H_{M} K_{X} H_{M}^{*}+\mathbf{I}\right)^{-1}=0 .
\end{gathered}
$$

One way of solving an algebraic Riccati [3] of the form

$$
\mathbf{0}=M_{21}+M_{22} A^{*}-A^{*} M_{11}-A^{*} M_{12} A^{*},
$$

is to look for invariant subspaces of

$$
M=\left(\begin{array}{ll}
M_{11} & M_{12} \\
M_{21} & M_{22}
\end{array}\right) .
$$

Here we have that $M$ is given by

$$
\begin{aligned}
M_{11}= & -\left(H_{M} K_{X} H_{M}^{*}+\mathbf{I}\right)^{-1} \\
M_{12}= & -\left(H_{M} K_{X} H_{M}^{*}+\mathbf{I}\right)^{-1} H_{M} K_{X} H_{E}^{*} \\
M_{21}= & H_{E} K_{X} H_{M}^{*}\left(H_{M} K_{X} H_{M}^{*}+\mathbf{I}\right)^{-1} \\
M_{22}= & -H_{E} K_{X} H_{E}^{*}-\mathbf{I}+ \\
& H_{E} K_{X} H_{M}^{*}\left(H_{M} K_{X} H_{M}^{*}+\mathbf{I}\right)^{-1} H_{M} K_{X} H_{E}^{*} .
\end{aligned}
$$

Set

$$
F=\left(\begin{array}{cc}
H_{M} K_{X} H_{M}^{*}+\mathbf{I}_{n_{M}} & 0 \\
0 & \mathbf{I}_{n_{E}}
\end{array}\right) .
$$

It is easy to see that $F(M+\mathbf{I})$ is given by

$$
\left[\begin{array}{c}
-H_{M} \\
-H_{E}+H_{E} K_{X} H_{M}^{*}\left(H_{M} K_{X} H_{M}^{*}+\mathbf{I}\right)^{-1} H_{M}
\end{array}\right] K_{X}\left[-H_{M}^{*} H_{E}^{*}\right]
$$

which implies that -1 is an eigenvalue of $M$. Thus a first invariant subspace is given by the eigenspace associated to -1 , which is the kernel of $M+\mathbf{I}$, or in other words, the subspace $\left(\begin{array}{ll}P^{T} & Q^{T}\end{array}\right)^{T}$ orthogonal to $\left(\begin{array}{lll}-K_{X} H_{M}^{*} & K_{X} H_{E}^{*}\end{array}\right)$.

We further rewrite $M$ as

$$
\begin{aligned}
& \left(\begin{array}{c}
-H_{M}\left(K_{X} H_{M}^{*} H_{M}+\mathbf{I}\right)^{-1} \\
-H_{E}+H_{E}\left(K_{X} H_{M}^{*} H_{M}+\mathbf{I}\right)^{-1} K_{X} H_{M}^{*} H_{M}
\end{array}\right) . \\
& \left(-K_{X} H_{M}^{*} \quad K_{X} H_{E}^{*}\right)-\mathbf{I}
\end{aligned}
$$

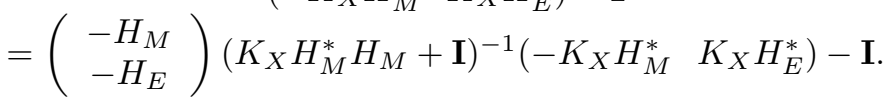

Thus, a Jordan basis of $M$ is given by

$$
\left(\begin{array}{cc}
H_{M} & P \\
H_{E} & Q
\end{array}\right)
$$

with $\left(\begin{array}{ll}P^{T} & Q^{T}\end{array}\right)^{T}$ orthogonal to $\left(\begin{array}{ll}-H_{M}^{*} & H_{E}^{*}\end{array}\right)$.

Finally, solutions of the Ricatti equation are given [3], in the general case, by:

$$
\tilde{A}^{*}=\left(\begin{array}{lll}
H_{E} V & Q W
\end{array}\right)\left(H_{M} V \quad P W\right)^{-1},
$$

where $W$ is an $n_{M} \times m$ matrix, $0 \leq m \leq n_{M}$, and $V$ is a $n_{M} \times n_{M}-m$ matrix, such that

$$
\left(\begin{array}{c}
H_{M} V \\
H_{E} V
\end{array}\right)
$$


is an invariant subspace of $M$. Note that $W$ can be chosen arbitrary since $\left(P^{T}, Q^{T}\right)^{T}$ is the eigenspace associated to -1 .

Proposition 7: Let $\tilde{K}_{X}$ be an optimal solution to the optimization problem

$$
\begin{array}{rc}
\max K_{X} & \min _{A} \tilde{I}(X ; Y \mid Z) \\
\text { s.t. } & K_{X} \succeq \mathbf{0}, \operatorname{Tr}\left(K_{X}\right)=P,
\end{array}
$$

where $\tilde{A}^{*}=\left(\begin{array}{lll}H_{E} V & Q W\end{array}\right)\left(\begin{array}{ll}H_{M} V & P W\end{array}\right)^{-1}$ is the optimal solution for the minimization over $A$. Then $\tilde{K}_{X}$ is low rank.

Proof: Note that $\tilde{I}(X ; Y \mid Z)$ can be written

$$
\log \operatorname{det}\left(\mathbf{I}+B K_{X}\right)-\log \operatorname{det}\left(\mathbf{I}+H_{E} K_{X} H_{E}^{*}\right),
$$

where

$$
B:=\left(H_{M}^{*}-H_{E}^{*} A^{*}\right)\left(\mathbf{I}-A A^{*}\right)^{-1}\left(H_{M}-A H_{E}\right)+H_{E}^{*} H_{E} .
$$

We now show that $B-H_{E}^{*} H_{E}$ is low rank by showing that $\left(H_{M}^{*}-H_{E}^{*} A^{*}\right)$ is low rank. Indeed, we have that $A^{*}=\left(\begin{array}{ll}H_{E} V & Q W\end{array}\right)\left(\begin{array}{ll}H_{M} V & P W\end{array}\right)^{-1}$. Therefore,

$$
\begin{aligned}
& H_{M}^{*}-H_{E}^{*} A^{*}=\left(\begin{array}{cc}
H_{M}^{*} & -H_{E}^{*}
\end{array}\right)\left(\begin{array}{c}
\mathbf{I} \\
A^{*}
\end{array}\right) \\
& =\left(\begin{array}{ll}
H_{M}^{*} & -H_{E}^{*}
\end{array}\right)\left(\begin{array}{cc}
H_{M} V & P W \\
H_{E} V & Q W
\end{array}\right)\left(\begin{array}{ll}
H_{M} V & P W)^{-1}
\end{array}\right.
\end{aligned}
$$

which, since $\left(P^{T} Q^{T}\right)^{T}$ is orthogonal to $\left(H_{M}^{*}-H_{E}^{*}\right)$ yields $H_{M}^{*}-H_{E}^{*} A^{*}=\left(\left(\begin{array}{lll}\left.H_{M}^{*} H_{M}-H_{E}^{*} H_{E}\right) V & \mathbf{0}\end{array}\right)\left(\begin{array}{ll}H_{M} V & P W\end{array}\right)^{-1}\right.$, which, as desired, is low rank.

Now, from Proposition 2, we know that either $B \succ H_{E}^{*} H_{E}$ and $\lambda>0$, or $B \prec H_{E}^{*} H_{E}$ and $\lambda<0$. This is a contradiction since $B \succeq H_{E}^{*} H_{E}$, yielding that $\tilde{K}_{X}$ is low rank.

Proposition 8: The rank of $\tilde{K}_{X}$ being $r<n$, that is $K_{X}=$ $U_{X} U_{X}^{*}$ with $U_{X}$ an $n \times r$ matrix, the optimal solution to

$$
\min _{A} \tilde{I}(X ; Y \mid Z)
$$

is given by

$$
\begin{gathered}
A^{*}=\left(\begin{array}{ll}
H_{E}\left(K_{X} H_{M}^{*} H_{M}+\mathbf{I}\right)^{-1} U_{X} V & Q W
\end{array}\right) \\
\left(H_{M}\left(K_{X} H_{M}^{*} H_{M}+\mathbf{I}\right)^{-1} U_{X} V \quad P W\right)^{-1} .
\end{gathered}
$$

Proof: The Jordan decomposition of $M$ is now given by

$$
M\left(\begin{array}{cc}
H_{M} & P \\
H_{E} & Q
\end{array}\right)=\left(\begin{array}{cc}
H_{M} & P \\
H_{E} & Q
\end{array}\right)\left(\begin{array}{cc}
J & \mathbf{0} \\
\mathbf{0} & -\mathbf{I}
\end{array}\right)
$$

where

$$
J=\left(\mathbf{I}+K_{X} H_{M}^{*} H_{M}\right)^{-1}\left(K_{X} H_{M}^{*}-K_{X} H_{E}^{*}\right)\left(\begin{array}{c}
H_{M} \\
H_{E}
\end{array}\right)-\mathbf{I} .
$$

Let us now look more carefully at $J$. We first notice that when $K_{X}$ is low rank, -1 is an eigenvalue. This is clear since

$$
J+\mathbf{I}=\left(\mathbf{I}+K_{X} H_{M}^{*} H_{M}\right)^{-1} K_{X}\left(\begin{array}{ll}
H_{M}^{*} & -H_{E}^{*}
\end{array}\right)\left(\begin{array}{c}
H_{M} \\
H_{E}
\end{array}\right)
$$

and $\operatorname{det}\left(K_{X}\right)=0$. Furthermore, since $K_{X}=U_{X} U_{X}^{*}$, we have

$$
J=\left(\mathbf{I}+K_{X} H_{M}^{*} H_{M}\right)^{-1} U_{X} U_{X}^{*}\left(\begin{array}{ll}
H_{M}^{*} & -H_{E}^{*}
\end{array}\right)\left(\begin{array}{c}
H_{M} \\
H_{E}
\end{array}\right)-\mathbf{I}
$$

and clearly $\left(\mathbf{I}+K_{X} H_{M}^{*} H_{M}\right)^{-1} U_{X}$ is an invariant subspace of $J$. A Jordan basis is thus given by

$$
P^{\prime}=\left(\begin{array}{ll}
\left(\mathbf{I}+K_{X} H_{M}^{*} H_{M}\right)^{-1} U_{X} \quad Q^{\prime}
\end{array}\right)
$$

where $Q^{\prime}$ is the eigenspace associated to -1 . This thus gives us a more precise Jordan basis for $M$ (as defined in (9)), namely

$$
\left[\begin{array}{cc}
H_{M} P^{\prime} & P \\
H_{E} P^{\prime} & Q
\end{array}\right]=\left[\begin{array}{ccc}
H_{M}\left(K_{X} H_{M}^{*} H_{M}+\mathbf{I}\right)^{-1} U_{X} & H_{M} Q^{\prime} & P \\
H_{E}\left(K_{X} H_{M}^{*} H_{M}+\mathbf{I}\right)^{-1} U_{X} & H_{E} Q^{\prime} & Q
\end{array}\right] .
$$

From this Jordan basis of $M$, we have that

$$
\begin{gathered}
A^{*}=\left(H_{E}\left(K_{X} H_{M}^{*} H_{M}+\mathbf{I}\right)^{-1} U_{X} V \quad Q W\right) \\
\left(H_{M}\left(K_{X} H_{M}^{*} H_{M}+\mathbf{I}\right)^{-1} U_{X} V \quad P W\right)^{-1}
\end{gathered}
$$

is a solution of the Ricatti equation, where $W$ is any $n_{M} \times$ $\left(n_{M}-r\right)$ matrix, and $V$ is any $r \times r$ matrix.

\section{The converse matches the achievability}

We can now conclude.

Proposition 9: Let

$$
\begin{gathered}
A^{*}=\left(H_{E}\left(K_{X} H_{M}^{*} H_{M}+\mathbf{I}\right)^{-1} U_{X} V \quad Q W\right) \\
\left(H_{M}\left(K_{X} H_{M}^{*} H_{M}+\mathbf{I}\right)^{-1} U_{X} V \quad P W\right)^{-1}
\end{gathered}
$$

be a solution of the Ricatti equation. Then

$\tilde{I}(X ; Y \mid Z)=\log \operatorname{det}\left(\mathbf{I}+H_{M} K_{X} H_{M}^{*}\right)-\log \operatorname{det}\left(\mathbf{I}+H_{E} K_{X} H_{E}^{*}\right)$.

Furthermore, there exists $V, W$ such that $\mathbf{I}-A A^{*} \succ \mathbf{0}$.

Now that the matrix $A^{*}$ is known explicitly, this can be checked by computation, which is omitted here by lack of space (see [18]).

\section{CONCLUSION}

In this paper, we considered the problem of computing the perfect secrecy capacity of a multiple antenna channel, based on a generalization of the wire-tap channel to a MIMO broadcast wire-tap channel. We proved that for an arbitrary number of transmit/receive antennas, the perfect secrecy capacity is the difference of the two capacities, the one of the legitimate user minus the one of the eavesdropper, after a suitable

optimization over the transmitter's input covariance matrix.

\section{ACKNOWLEDGEMENT}

This work was supported in part by NSF grant CCF0729203, by Caltech's Lee Center for Advanced Networking and by a grant from the David and Lucille Packard Foundation.

\section{REFERENCES}

[1] J. Barros and M. R. D. Rodrigues, "Secrecy Capacity of Wireless Channels", IEEE International Symposium on Inf. Theory, Seattle, 2006.

[2] M. Bloch, J. Barros, M. R. D. Rodrigues, S. W. McLaughlin, "Wireless Information-Theoretic Security - Part I: Theoretical Aspects". Submitted to IEEE Transactions on Information Theory, Special Issue on Information-Theoretic Security, November 2006

[3] G. Freiling, "A Survey on Nonsymmetric Ricatti Equations", Lin. Algebra and its Appl., 251-252, 2002.

[4] P. Gopala, L. Lai, and H. El Gamal, "On the Secrecy Capacity of Fading Channels", submitted to IEEE Transactions on Inf. Theory, Oct. 2006

[5] A. O. Hero, "Secure Space-Time Communication," , IEEE Trans. on Info Theory, Vol. 49, No. 12, pp. 1-16, Dec. 2003.

[6] T. Kailath, A.H. Sayed and B. Hassibi, "Linear Estimation", PrenticeHall, 2000. 
[7] A. Khisti, G. Wornell, A. Wiesel, Y. Eldar, "On the Gaussian MIMO Wiretap Channel", in IEEE Int. Symposium on Inf. Theory, Nice, 2007.

[8] A. Khisti, G. Wornell, "Secure Transmission with Multiple Antennas: The MISOME Wiretap Channel", Submitted, August 2007.

[9] S.K. Leung-Yan-Cheong, M.E. Hellman, "The Gaussian Wire-Tap Channel”, IEEE Trans. on Information Theory, vol. 24, July 1978.

[10] Z. Li, R. Yates, W. Trappe,"Secrecy capacity of independent parallel channels", in Allerton conference, 2006.

[11] Z. Li, W. Trappe, R. Yates, "Secret communication via multi-antenna transmission", in Conference on Information Sciences and Systems (CISS), March 2007.

[12] Y. Liang, H. V. Poor, "Secure Communication over Fading Channels", in Proc. of Allerton, 2006.

[13] Y. Liang, H. V. Poor, Shlomo Shamai (Shitz), "Secure Communication over Fading Channels", Submitted to IEEE Transactions on Inf. Theory, Special Issue on Information Theoretic Security, November 2006

[14] R. Liu, H. V. Poor, "Multiple Antenna Secure Broadcast over Wireless Networks", First International Workshop on Information Theory for Sensor Networks, Santa Fe, 2007.

[15] R. Liu, H. V. Poor, "Secrecy Capacity Region of a Multi-Antenna Gaussian Broadcast Channel with Confidential Messages", submitted to IEEE Transactions on Information Theory, (arXiv:0709.4671)

[16] T. Liu, Shlomo Shamai (Shitz), "A Note on the Secrecy Capacity of the Multi-antenna Wiretap Channel",

submitted to IEEE Trans. on Information Theory, (arxiv.org/pdf/0710/4105.pdf).

[17] F. Oggier, B. Hassibi, "The Secrecy Capacity of the 2x2 MIMO Wiretap Channel", in Allerton Conference, September 2007.

[18] F. Oggier, B. Hassibi, "The Secrecy Capacity of the MIMO Wiretap Channel", submitted.

[19] P. Parada, R. Blahut,"Secrecy capacity of SIMO and slow fading channels," in IEEE Int. Symposium on Inf. Theory, Adelaide, 2005.

[20] S. Shafiee, S. Ulukus, "Achievable Rates in Gaussian MISO Channels with Secrecy Constraints", in IEEE Int. Symp. on Inf. Theory, Nice, 2007.

[21] S. Shafiee, N. Liu and S. Ulukus, Towards the Secrecy Capacity of the Gaussian MIMO Wire-tap Channel: The 2-2-1 Channel, submitted to IEEE Trans. on Information Theory, September 2007.

[22] A.D. Wyner, "The wire-tap channel," Bell. Syst. Tech. J., vol. 54, October 1975. 